\title{
Methodological problems in QFD and directions for future development
}

\author{
Ibo van de Poel
}

Received: 20 September 2005/Revised: 5 March 2007 / Accepted: 19 March 2007/Published online: 25 April 2007

(C) Springer-Verlag London Limited 2007

\begin{abstract}
Quality function deployment (QFD) is a popular tool for product development in industry. QFD aims at setting targets for product characteristics so that products optimally meet customer demands. In this article, the focus is not on the actual effects of QFD but on more fundamental possibilities and limitations of QFD. In particular, I will discuss a number of methodological problems in QFD. One of the most disturbing methodological problems is the impossibility of translating individual into collective customer demands and the impossibility of translating customer demands into engineering characteristics without violating one or more very reasonable conditions. These problems are due to Arrow's Impossibility Theorem. I discuss whether a number of alternative QFD approaches are helpful in overcoming these methodological problems and suggest directions for the further development of QFD and for research.
\end{abstract}

Keywords Quality function deployment · Product development · Methodology · Arrow's Impossibility Theorem · Market segments · Optimization

\footnotetext{
An earlier version of this paper was presented at the meeting of the Society for Philosophy and Technology (SPT), 7, 8 and 9 July 2003 in Park City, Utah, USA. I would like to thank Maarten Franssen for comments on an earlier version.
}

I. van de Poel $(\square)$

Department of Philosophy, School of Technology, Policy and Management, Delft University of Technology, P.O. Box 5015, 2600 GA Delft, The Netherlands e-mail: i.r.vandepoel@tbm.tudelft.nl

\section{Introduction}

Quality function deployment (QFD) was originally developed in Japan in the late 1960s. It is now widely used not only in Japan, but also in Europe and the United States. The introduction of QFD, and other quality methods, in especially the USA, was a response to the growing success of the Japanese industry during the 1970s. QFD was seen as an important tool to improve quality, to reduce development and other pre-production costs, to increase organization capabilities and-all in all-to make industry more competitive. Apart from such business goals, QFD has been heralded as a means for the development of products that better fulfil users' needs. Some have even claimed that this is the main purpose of QFD (e.g. Hauser and Clausing 1988, p. 63; Bergquist and Abeysekera 1996, p. 273).

A main goal of QFD is to translate customer demands into target values for the engineering characteristics of a product. By systematically and quantitatively employing the relationship between customer demands and engineering characteristics, those engineering characteristics that are most promising for improving customer satisfaction can be selected and target values can be set. In this way, QFD results in a more systematic attention for customer demands in the design and development process, or at least that is claimed. As Fung et al. (2003) wrote in a recent article in this journal: "Being an important business goal, customer satisfaction is a growing concern and prerequisite towards effective competitiveness" (Fung et al. 2003, p. 1).

The use of QFD and related methods is to result in "achieving maximized overall customer satisfaction", (Fung et al. 2003, p. 1). Also Franceschini and Rossetto see the maximisation of customer satisfaction as the main goal of QFD: “[p]roduct designers need to know how to make tradeoffs in the selection of design features which result in 
Fig. 1 House of Quality

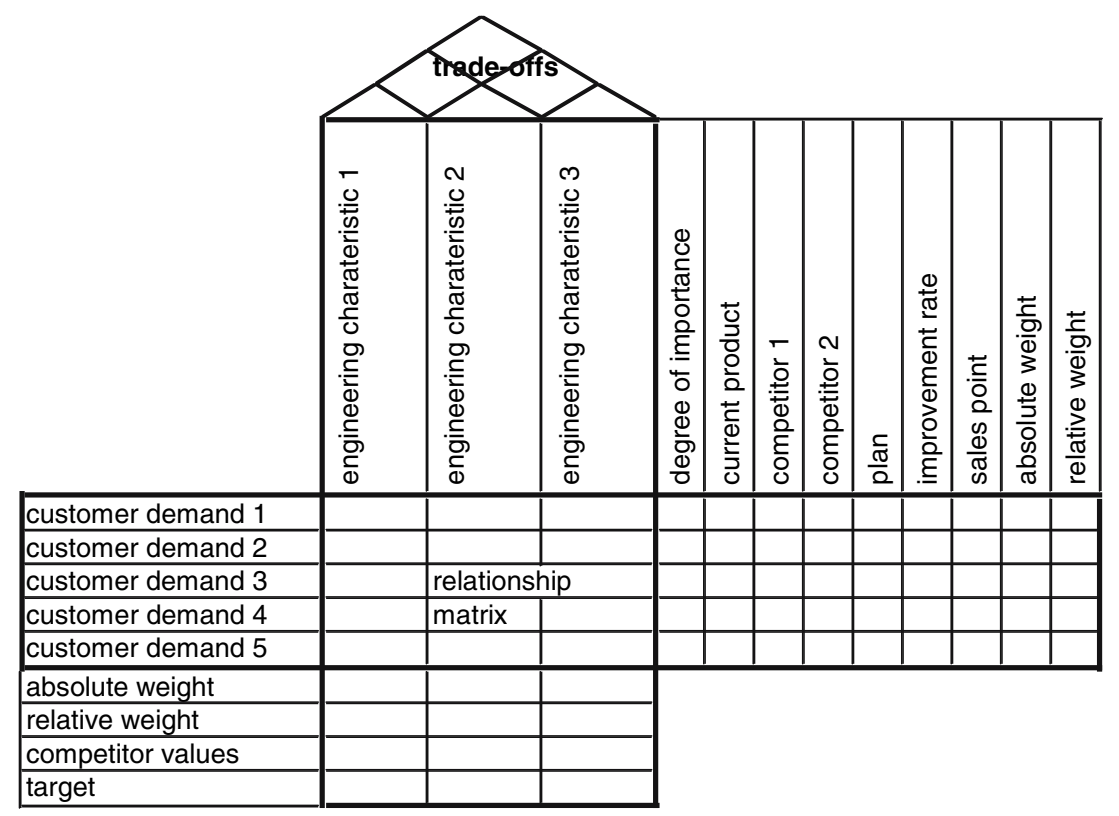

the highest level of customer satisfaction" (Franceschini and Rossetto 1995, p. 270).

In this article, the focus is not on the actual effects of QFD on industrial and engineering practice, but on methodological issues in QFD. One might argue that such methodological problems do not hamper the success of QFD in actual practice. Even if this were true, some of the methodological problems imply that it is hard to determine whether QFD indeed leads to "'better", products, as is often claimed.

I start with a brief description of the QFD method in the next section. In Sects. 3 and 4, the methodological problems of QFD are discussed. In Sect. 5, some solutions to these methodological problems that have been proposed in the literature on product development are critically discussed. I show that none of these entirely solves all methodological problems in QFD, although some suggest interesting directions for the further development of QFD that might eventually overcome, or at least diminish, the methodological problems. In the final section, I draw conclusions and discuss possible directions for further research.

\section{What is QFD?}

A central element in QFD is the so-called "House of Quality", (Fig. 1). ${ }^{1}$ This House of Quality relates customer

\footnotetext{
${ }^{1}$ QFD is not one method but an amalgam of similar methods and tools. Some authors have argued that QFD is not so much a method as well as an organizational principle that has to pervade the complete organization.
}

demands to engineering characteristics. ${ }^{2}$ The idea is that in this way desires of customers can be translated into target values for the engineering characteristics and in priorities for improving certain engineering characteristics.

Filling in the House of Quality starts with listing the customer demands in the rows in the central part of the house. Subsequently, the degree of importance of the customer demands is filled in. The score of the own existing product and that of competitors with respects to the customer demands are then listed, usually on an integer scale from 1 to 5 . On the basis of this competitive benchmarking and strategic considerations, the company plan for each customer demand is chosen, again on an integer scale from 1 to 5 . The rate of improvement is calculated by dividing the company plan by the current company score. ${ }^{3}$

Next, sales points are set for customer demands that are expected to influence sales more than average. Sales points usually take the values $1.5,1.2$ or 1 . The absolute weight of the customer demands is calculated by multiplying the degree of importance with the rate of improvement and the sales point (King 1989; Akao 1990).

The next step is relating the customer demands to the engineering characteristics. To achieve this, first the engi-

\footnotetext{
${ }^{2}$ Different authors use somewhat different terminology like customer requirements, customer attributes, demanded quality and customer needs instead of customer demands and quality characteristics, technical attributes, design parameters, product technical requirements and product characteristics instead of engineering characteristics (Hauser and Clausing 1988; King 1989; Akao 1990; Shullito 1994; Bergquist and Abeysekera 1996; Govers 1996).

${ }^{3}$ It might be argued, however, that since both the company plan and the current company score are measured on an ordinal scale (expressed in the integers $1,2,3,4,5)$ this division is not allowed because ordinal scales do not allow for this arithmetical operation.
} 
Table 1 Typical variables and calculations used in QFD

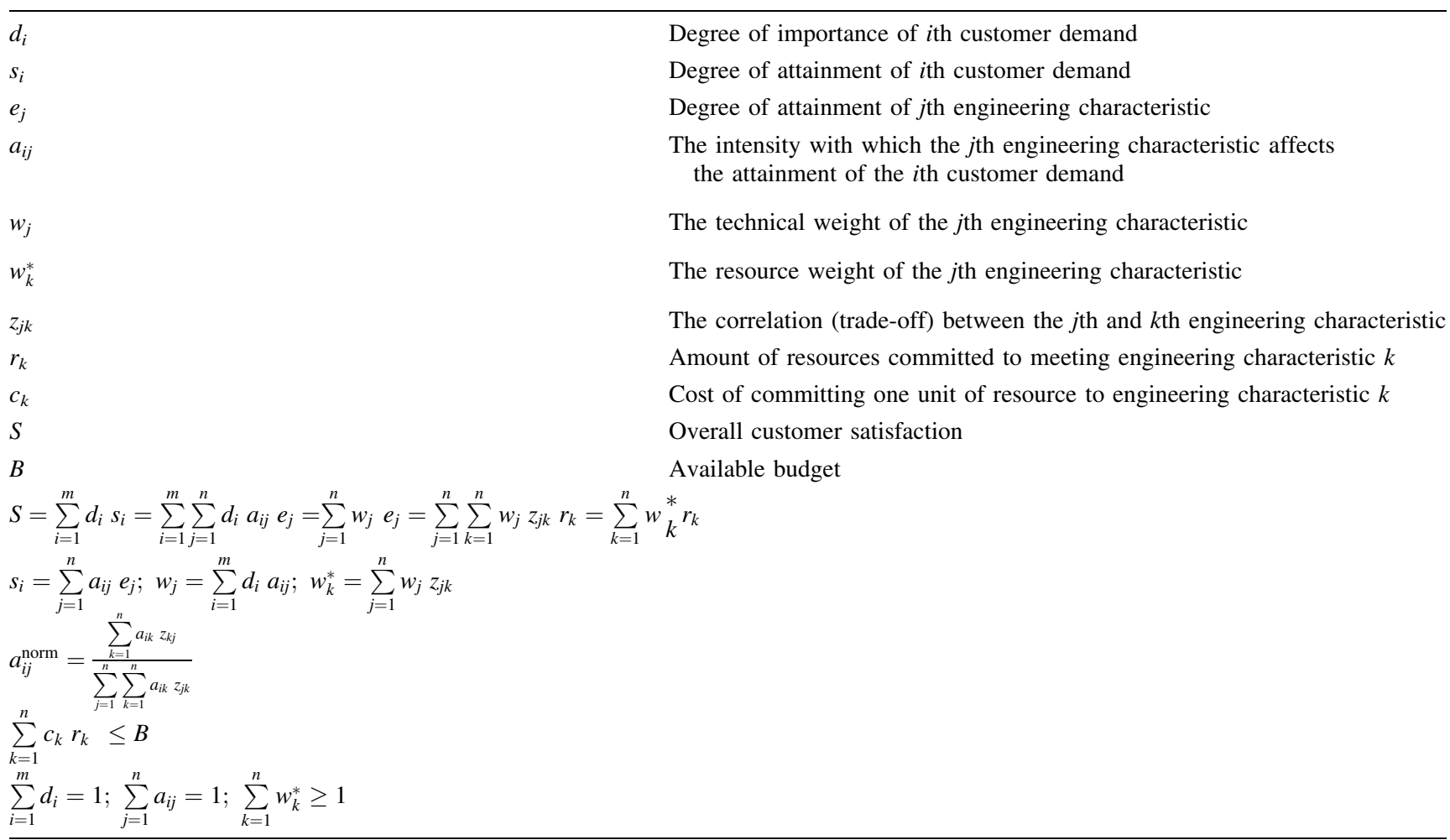

neering characteristics are listed in the columns in the central part of the House of Quality. Next the relationship matrix is filled in, using symbols like () (strong correlation), $\bigcirc$ (moderate correlation) and $\Delta$ (weak correlation), which are presumed to correspond with numerical values like, for example, 9, 3 and 1. On the basis of the weighted customer demands and the relationship matrix the relative importance of the engineering characteristics is calculated (See Table 1). The values of the engineering characteristics for the current product and those of competitors are listed, and targets for the engineering characteristics may be set. As a final step, the trade-offs between the engineering characteristics are listed in the roof of the House of Quality. ${ }^{4}$ Usually five types of relations between engineering characteristics are used: strong positive, weak positive, no relation, weak negative, strong negative.

The House of Quality thus gives insight in the relative importance of the engineering characteristics based on the customer demands. This relative importance may be used to set priorities in further design and development efforts or to select among different conceptual designs. ${ }^{5}$ By making a

\footnotetext{
${ }^{4}$ This step is absent in Akao (1990) and King (1989).

5 Typically, however, King (1989) presents different variants of socalled Pugh Charts (Pugh 1991) as method for concept selection in which the relative weights of the customer demands are engineering characteristics do not play a role.
}

number of further charts, the relative importance of customer demands or engineering characteristics can also be translated into relative weights for certain functions, mechanisms, parts, process steps, failure mechanisms and in setting priorities for these and for cost reduction (King 1989; Akao 1990).

Also target values for the engineering characteristics can be determined. Apart from the relative weights of the engineering characteristics, estimates about what is technically feasible against what costs and efforts, and strategic considerations at the company level do play a role in setting targets. In the initial method, setting targets was left to the discretion of the engineers on the basis of the filled in House of Quality.

A quantitative approach to setting targets has been proposed by Bode and Fung (1998), based on an earlier proposal by Wasserman (1993). Later, sophistications to this approach have been developed (Fung et al. 1998, 2002, 2003, Tang et al. 2002). For the moment I restrict my self to the approach proposed by Bode and Fung because this is enough to discuss some of the methodological issues with respect to setting targets. I will later discuss whether the more sophisticated approaches alleviate the methodological problems or not.

In the approach of Bode and Fung, targets are set by taking into account costs considerations. The idea is that without cost considerations, all engineering characteristics 
Fig. 2 Pencil example (based on Bode and Fung 1998; Fung et al. 2002)

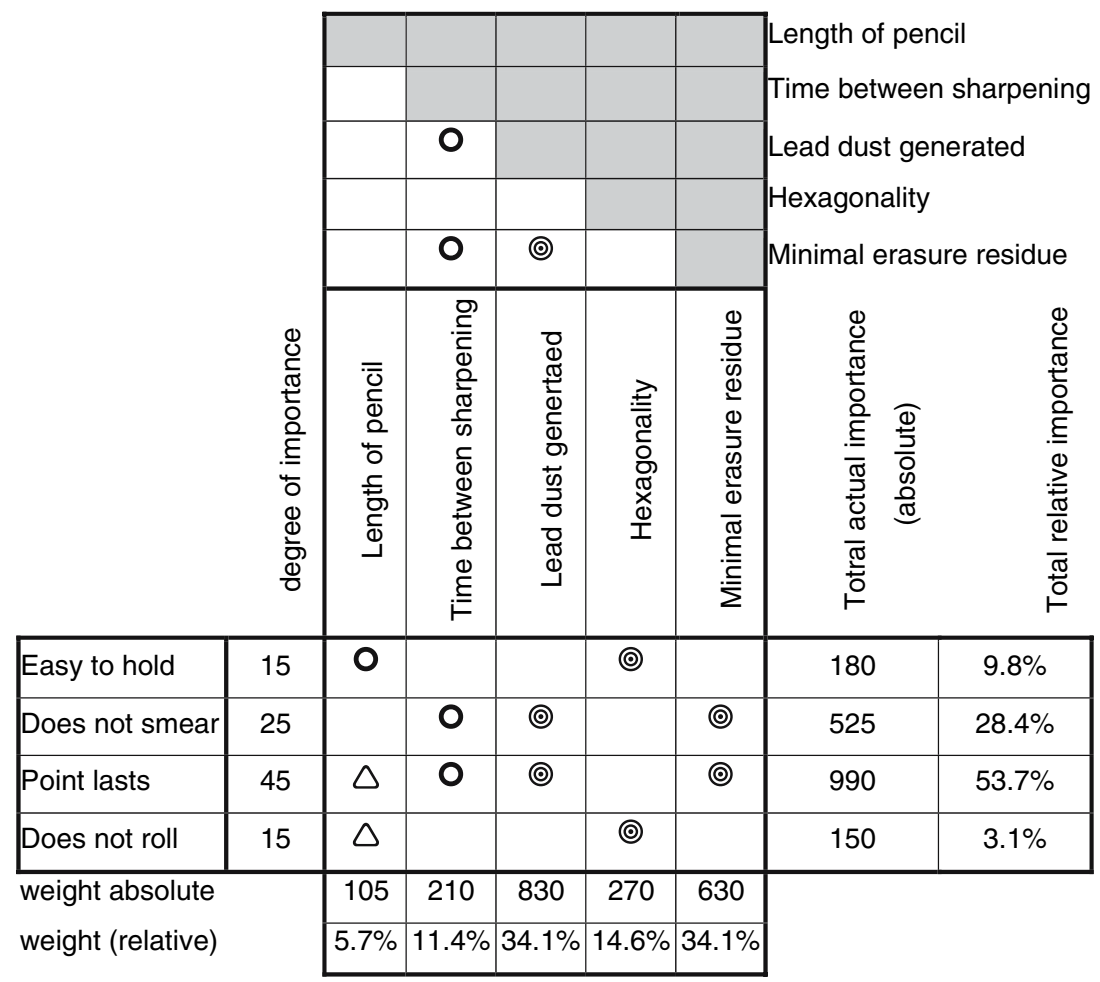

are to be met maximally, i.e. by a factor 1 . Cost considerations are then used to determine the desirable factor by which each engineering characteristic is to be met given the available budget. This is done by maximising customer satisfaction given a budget constraint. In doing so, Bode and Fung take into account that the engineering characteristics are related to each other by a correlation factor $z_{j k}$. This is required to correct for the fact that by committing resources to engineering characteristic $j$, one also influences the attainment of engineering characteristic $k$.

Figure 2 shows an example of the QFD approach for the (re)design of a pencil. The numerical values in the example are based on Bode and Fung (1998) and Fung et al. (2002). In the rows, customer demands, like "easy to hold," are listed. In the columns the engineering characteristics, like " 'time between sharpening,', are listed. The weight of the engineering characteristics can now be easily calculated by associating the symbols $\odot, \bigcirc$ and with the strengths 9,3 and 1. (For the relevant variables and formulas, see Table 1). This way of calculating, however, results in a distortion of the degree of importance of the customer demands as shown in the last two columns of Fig. 2: the actual importance rating, indicated in the last two columns, is different from the intended importance, indicated in the first column. This distortion can be corrected by normalizing the relationship matrix so that the sum of the correlations $a_{i j}$ in each row is 1. Wasserman (1993) has proposed an extension to this approach in which also the correlation between the engineering characteristics is taken into account. In this approach a normalized $a_{i j}$ is calculated as follows:

$$
a_{i j}^{\text {norm }}=\frac{\sum_{k=1}^{n} a_{i k} z_{k j}}{\sum_{j=1}^{n} \sum_{k=1}^{n} a_{i k} z_{j k}} .
$$

The results of this normalization are shown in Fig. 3.

The trade-offs between the engineering characteristics are shown in the roof of Figs. 2 and $3 .{ }^{6}$ The target values for the engineering characteristics can be calculated by maximising customer satisfaction $S$ :

$S=\sum_{j=1}^{n} w_{j} e_{j}=\sum_{j=1}^{n} \sum_{k=1}^{n} w_{j} z_{j k} r_{k}$.

Under the budget constraint:

$\sum_{k=1}^{n} c_{k} r_{k} \leq B$

\footnotetext{
${ }^{6}$ For the trade-off matrix between the engineering characteristics the strengths $18,9,3$ and 1 are used. This matrix is "normalized" by associating 18 with a relative strength of 1 . Note that in this case the sum of the correspondence values in a row can be larger than 1 . The idea behind this is that by achieving, say, engineering characteristic $j$, one gets an amount of engineering characteristic $k$ for free.
} 
Fig. 3 Pencil example with normalized relationship matrix

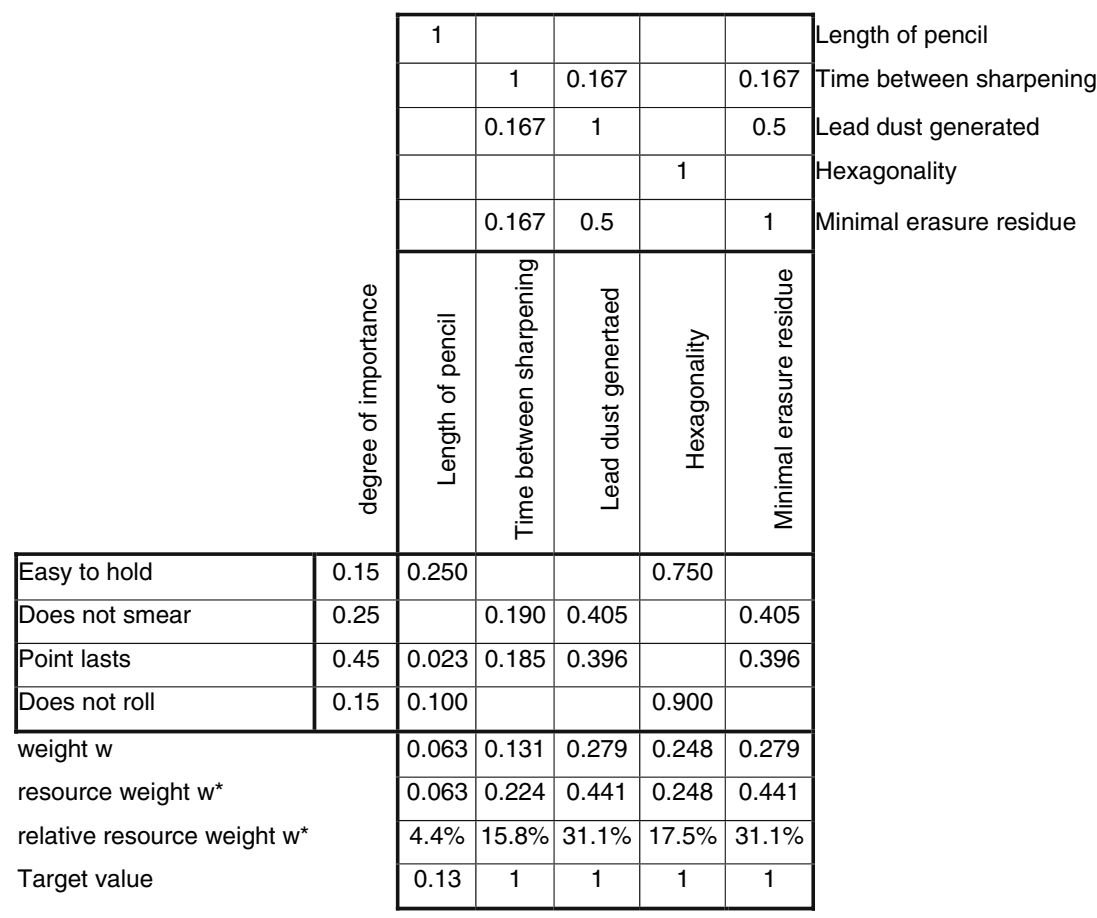

Figure 3 shows the resulting target values, based on the method proposed by Bode and Fung (1998) for solving the above linear model, as they are given in Fung et al. (2002, p. 596).

To summarize: a main aim of QFD is to translate customer demand into target values for engineering characteristics. Roughly, this translation is made in two steps:

- The formulation of collective degrees of importance for the customer demands on the basis of individual customer demands.

- The translation of the relative importance of customer demands into the relative importance of engineering characteristics, and the formulation of target values for these engineering characteristics.

In the next two sections, I will critically assess both steps as they are now usually carried out in QFD and show that both steps are beset with methodological problems.

\section{The transition from individual to collective customer demands}

A central part of QFD is the listing of the relevant customer demands and the determination of their relative importance. This step is in fact crucial if QFD is to lead to more consumer-oriented product development and design. In the original QFD method-apart from the individual customer demands-also the rate of improvement and the sales point play a role in setting the relative importance of the customer demands. In this section, I leave aside such "company considerations" as is in fact often done in the literature (e.g. Hauser and Clausing 1988; Franceschini and Rossetto 1995; Govers 1996; Bode and Fung 1998; Park and Kim 1998; Shen et al. 1999; Vairaktarakis 1999; Kim et al. 2000; Tang et al. 2002; Fung et al. 2003).

Although the literature on QFD is not entirely clear on how customer demands are to be collected and how their relative importance is to be determined, the central idea is that a representative number of customers are asked for their demands and the relative importance of these demands. ${ }^{7}$ These individual answers are then aggregated, resulting in a list of collective demands. Aggregation often occurs by taking the weighted average of the various individual customers or by using the Analytic Hierarchical Process (AHP) (Bergquist and Abeysekera 1996; Park and Kim 1998; Xie et al. 1998). There are at least three reasons why this procedure is methodologically problematic:

- Customer demands are product dependent (Sect. 3.1).

- Customer demands cannot always be represented by a linear additive value function (Sect. 3.2).

- Individual customer demands cannot be aggregated into a collective customer demands ordering without violating a number of very reasonable conditions (Sect. 3.3).

\footnotetext{
${ }^{7}$ It seems that in practice it often are the designers who determine the relative weight of the customer demands (Vairaktarakis 1999). Most proposals for QFD, however, seem to presuppose that the relative weight is to be based on customer preferences, which is in fact more obvious if one wants to maximize customer satisfaction.
} 


\subsection{Customer demands are product dependent}

Customers are usually not able to voice their demands with respect to products they do not know or have no experience with. This problem has in fact been recognized in the literature on QFD: customers are hardly able to voice their demands with respect to new products. Therefore, it has been advised in the QFD literature to focus on the improvement of existing products (Sarlemijn and Boddendijk 1995; Vairaktarakis 1999). Nevertheless, QFD has also been used to develop new products (Bergquist and Abeysekera 1996, p. 270). The above also implies that the choice for a certain product, and in fact also the choice for a certain group of customers, precedes the use of QFD.

\subsection{The representation of customer demands}

QFD presupposes that overall customer satisfaction $(S)$ can be represented by a linear additive value function of the degree of attainment $\left(s_{i}\right)$ of the individual customer demands:

$S=\sum_{i=1}^{m} d_{i} s_{i}$

How are we to interpret this function $S$ ? The most plausible interpretation, I think, is to interpret $S$ as a multiattribute value function that corresponds with certain customer preferences over options. Each option consists of $m$ attributes $A_{1}, \ldots, A_{m}$ that correspond with the user demands. Different options are characterized by different bundles of values $x_{1}, \ldots, x_{m}$ for these attributes. These values can be chosen in such a way that they correspond to the degree of attainment of the user demands $s_{i}, \ldots, s_{m}$. Now the fact that a customer prefers an option $\mathbf{x}\left(x_{1}, x_{2}, \ldots, x_{m}\right)$ over another option $\mathbf{y}\left(y_{1}, y_{2}, \ldots, y_{m}\right)$ corresponds with a value function $v$ so that $v\left(x_{1}, x_{2}, \ldots, x_{m}\right)>v\left(y_{1}, y_{2}, \ldots, y_{m}\right)$ if certain conditions are met (see, e.g. French 1988, pp. 74-82, 103-106). Thus, given that $d_{i}$ is fixed for a given $i$, the value function $S$ corresponds with the preferences of a customer as follows:

$\sum_{i=1}^{m} d_{i} x_{i} \geq \sum_{i=1}^{m} d_{i} y_{i} \Leftrightarrow x\left(x_{i}, \ldots, x_{n}\right) \succsim y\left(y_{i}, \ldots, y_{n}\right)$

In QFD, $S$ is represented as an additive linear value function with weighing factors $d_{1}, \ldots, d_{m}$. This representation is valid if three axioms are met (French 1988, p. 130):

1. Weak ordering: $\succsim$ is a weak order. This, among other things, implies that customers can always rank two options or are indifferent between them. Since this should be true for all combinations of values for $s_{1}, \ldots, s_{m}$, it means that customers should be able to rank

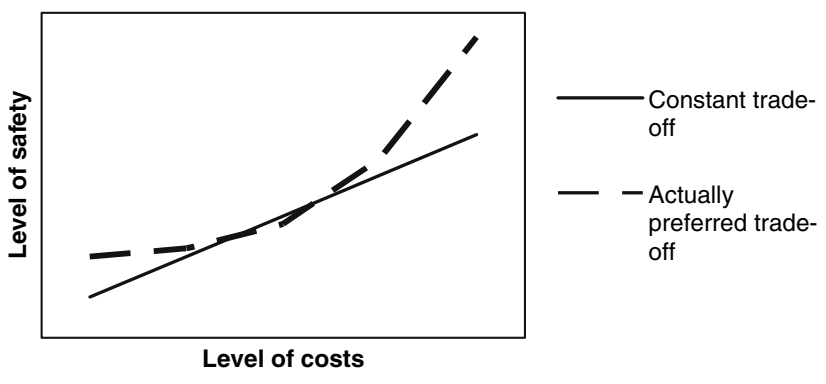

Fig. 4 Trade-off between safety and costs

options they do not know or even options consisting of combinations of values for $s_{1}, \ldots, s_{m}$ that are not feasible. ${ }^{8}$ This axiom also implies transitivity: if a customer prefers option $a$ over option $b$ and option $b$ over option $c$, she should also prefer option $a$ over option $c$. It is conceivable that in many cases the preferences of a customer do not meet these conditions.

2. Constant relative trade-offs: the trade-offs between the attributes are constant and fixed by the values of $d_{i}$. This is a very strong condition. I will discuss its (im)plausibility below.

3. Monotonicity, which implies that options $\mathbf{a}\left(a_{1}\right.$, $\left.a_{2}, \ldots, a_{m}\right)$ exist that are positively valued and that for any option $\mathbf{b}\left(b_{1}, b_{2}, \ldots, b_{m}\right)$, and any $\lambda>0, \mathbf{b}+\lambda \mathbf{a} \succ \mathbf{b}$. This axiom assumes that "more is better". Often, however, a customer demand will have an optimal value and more of it might add nothing or make it worse. Nevertheless, in such cases it is often possible to reformulate the original customer demand so that more is indeed better and the monotonicity condition is met. Sometimes, this can, for example, be achieved by replacing $s_{i}$ by $1 /\left(o_{i}-s_{i}\right)$ where $o_{i}$ is the optimal value for $s_{i}$. Note that for $s_{i} \rightarrow o_{i}, 1 /\left(o_{i}-s_{i}\right) \rightarrow \infty$.

Of these conditions, the assumption of constant relative tradeoffs is the strongest. It supposes that the trade-off ratio between two different customer demands is constant. In general, this is not a plausible assumption. An example might illustrate the point (Fig. 4). Suppose that there are two relevant customer demands with respect to cars: safety and costs. Now in the "House of Quality", the relative importance of the demands has to be indicated; for example

\footnotetext{
${ }^{8}$ One reason for the infeasibility of certain options may the be that the attributes are not independently realizable, as often is the case in engineering (cf. Franssen 2005, p. 52). In fact, the use of the relationship matrix in QFD is an indication that customer demands are often not independently realizable because engineering characteristics have an impact on more than one customer demand, so that some combinations of the customers demands may be impossible to realize. Lack of independent realizability is a reason to doubt whether $\succsim$ is well-defined for all combinations of values for $s_{1}, \ldots, s_{m}$ (Krantz et al. 1971 , p. 247). Note that, in that case, $\succsim$ might still be well-defined for a finite number of combinations of $s_{1}, \ldots, s_{m}$.
} 
that costs are considered twice as important as safety. It is, however, unlikely that people's judgement about the relative importance of safety versus costs is completely independent of the achieved levels of safety and costs. It seems likely that many people will consider safety the most important consideration up to a certain level of safety and will consider cost more important above that level. When this is the case, QFD might result in a misrepresentation of the preferences of customers.

Proponents of QFD might react to this objection in two ways. One reaction would be to argue that QFD usually focuses on limited improvements of existing products. It might be argued that in the range of products actually considered the customer demands can adequately represented by an additive value function (cf. Fig. 4). This might be true at least for some products and for some improvements. It is, however, something that needs to be checked, not something that can be taken for granted as happens in most of the QFD literature.

Another reply would be to adapt the QFD method and to represent customer preferences not as a linear additive value function, but in another, mathematically more complex, way. One attractive representation would be a nonlinear additive value function. For such a representation to be possible, the customers demands-corresponding with the attributes $A_{1}, \ldots, A_{m}$ - have to be preferentially independent (Keeney and Raiffa 1993, p. 111). ${ }^{9}$ A set of attributes $X$ is preferentially independent of its complement $Y$ if, and only if, the preference relation between options that differ only in the attributes $X$ and are similar in the attributes $Y$, does not depend on the exact values of the attributes $Y$. For example, the preferred cost for a car should not depend on its safety, and the desirable degree to which the point of a pencil lasts should not depend on how easy the pencil is to hold.

\subsection{From individual to collective customer demands}

We have seen that under certain, albeit rather strong, conditions customer preferences can be represented as a linear additive value function. This representation presupposes that the customer is able to weakly order any pair of options (axiom 1 in Sect. 3.2). One could, for example, ask a customer to make comparisons between a range of options and, on that basis, construct a value function representing the preferences of the customer. Under certain conditions, this value function has the form of a linear additive value function.

This procedure, however, does not work for a group of customers who have at least some conflicting preferences.

\footnotetext{
${ }^{9}$ For an alternative condition for only a finite number of attribute values, see Fishburn (1970, Chapt. 4).
}

In such cases, we cannot ask the group for its preferences. Rather, we are confronted with the problem of how to translate preferences of individual customers into collective preferences of the entire group of customers. I think that the best way to present this problem here is to cast it in terms of Arrow's Impossibility Theorem. This theorem, proved by Arrow (1950), shows that-in cases of at least two individuals and at least three options-it is impossible to find a function or decision procedure that meets a number of minimally reasonable conditions to translate individual into collective preferences. These minimal conditions are: ${ }^{10}$

- Collective rationality: This condition implies that the collective preference ordering must be complete and transitive. A preference ordering is complete if all alternatives are ordered by it. Transitivity requires that if $\mathrm{A}$ is ordered over $\mathrm{B}$ and $\mathrm{B}$ is ordered over $\mathrm{C}, \mathrm{A}$ is also ordered over C.

- Unrestricted domain: This condition implies that there are no restrictions with respect to how an individual orders the alternatives, apart from conditions of completeness and transitivity for the individual preference orderings.

- Pareto principle: This condition implies that if everyone prefers $\mathrm{A}$ to $\mathrm{B}$, the collective preference ordering should order A over B.

- Independence of irrelevant alternatives: The ordering of alternative A relative to alternative B may not depend on the inclusion or exclusion of a third alternative in the set of alternatives.

- Absence of a dictator: This condition implies that there is no individual whose preferences determine the collective preference.

Arrow's theorem means that no general procedure exist to translate individual preferences into a collective preference ordering unless one is willing to breach one of the above-mentioned conditions. ${ }^{11}$ The absence of a collective preference ordering over the options implies that these preferences cannot be represented by a value function (French 1988, p. 75). Therefore it is, in general, not possible to represent collective customer preferences by a value function $S$ as in done in QFD. It should be stressed that Arrow's Impossibility Theorem only shows that such a

\footnotetext{
${ }^{10}$ The requirements given are somewhat weaker than those originally formulated by Arrow. See, e.g. Sen (1970). See also Franssen (2005).

11 Arrow proved his theorem for ordinal individual preference orderings (i.e. preference ordering that order the alternatives with respect to their importance but contain no information with respect to their relative importance). Later, theorems comparable to Arrow's theorem have been proved for cardinal individual preference orderings, which also contain information about the relative importance of alternatives on an interval scale (e.g. Sen 1970).
} 
representation is not possible in general; it might still be possible in specific cases; for example if all customers have the same preferences.

One important reason for Arrow's theorem is that interpersonal comparison of preferences is not deemed possible (French 1988, pp. 288-298). This means-among other things - that it is not meaningful to add up the relative weights of the different customer demands given by the individual customers and to derive-by taking the mean or otherwise-the collective relative weights of the customer demands.

This fundamental difficulty in translating individual customer preferences into a collective preference ordering may be a reason why so much of the literature on QFD is silent on how individual preferences are to be translated into a collective preference ordering. Many publications simply presuppose that the relative importance of the customer demands is given or has been obtained in a further unspecified way. If it is mentioned how individual customer preferences have been attained and how these have been translated into a collective ordering of the customer demands, no justification is given for the chosen method (e.g. Bergquist and Abeysekera 1996; Park and Kim 1998).

\section{Rating engineering characteristics and setting tartgets}

A second important step in QFD is the translation of the relative importance of customer demands into the relative importance of the engineering characteristics, and the setting of target values for these characteristics. Again, three methodological problems are attached to this procedure:

- The correlation between customer demands and engineering characteristics is not always non-negative and constant (Sect. 4.1).

- The relative importance of customer demands cannot be uniformly translated into a relative importance for engineering characteristics (Sect. 4.2).

- The meaning of target values is unclear or disputable (Sect. 4.3).

\subsection{The relationship matrix}

In the relationship matrix in the House of Quality the correlation between customer demands and engineering characteristics in indicated. QFD presupposes that the (relative) weight of the engineering characteristics $\left(w_{j}\right)$ can be expressed as a linear additive function of the (relative) importance of the customer demands: $w_{j}=\sum_{i=1}^{m} d_{i} a_{i j}$.

In this formula, $a_{i j}$ is the correlation between the attainment of the $j$ th engineering characteristic and the attainment of the $i$ th customer demand. QFD presupposes that $a_{i j}$ is always non-negative (see Sect. 2). This assumption can cause problems, as can be illustrated with the following example. One of the customer demands for cars is "fuel consumption". This can, for example, be achieved through the engineering characteristic "weight of the car', i.e. lighter cars have lower fuel consumption ceteris paribus. However, lighter cars get a higher relative acceleration in collisions with heavier cars and, therefore, are more dangerous to the driver and passengers. ${ }^{12}$ So, while the engineering characteristic "weight of the car", correlates positively with the customer demand "safety for the driver and for the passengers", it correlated negatively with the customer demand "fuel consumption". There are two ways to adapt the original QFD approach to deal with this type of situation.

One way is to take the absolute value of the correlation between the $j$ th engineering characteristic and the attainment $i$ th customer demand in determining $a_{i j}$. The effect would be that an engineering characteristic that correlates positively with one customer demand and negatively with another becomes overall more important. This might be considered desirable in as far as that both the positive and the negative correlation are an indication that this is indeed an important engineering characteristic. The disadvantage, however, is that it becomes unclear what the optimal value of the engineering characteristic is or even in what direction the optimal value should be sought-in this case: more weight or less weight — while the target setting approach in QFD that I discussed in Sect. 2 presupposes that it is clear what the optimal value of an engineering characteristic is. This approach thus increases the methodological problem that will be discussed in Sect. 4.3: target values are unclear or meaningless.

Another approach would be to introduce negative $a_{i j}$ for situations in which the correlation between the $j$ th engineering characteristic and the attainment of the $i$ th customer demand is negative. ${ }^{13}$ One effect of introducing negative $a_{i j}$ might be that the overall importance of some

\footnotetext{
12 This example is inspired by the design of an lightweight vehicle (Dutch EVO) discussed in Van Gorp (2005).

${ }^{13}$ If an engineering characteristic has only negative correlations with customer demands, it is probably better to reformulate it, so that is has a positive correlation with customer demands. The point here is that engineering characteristics that have at least some positive correlations with some user demands might have negative correlations with other user demands.
} 
engineering characteristics becomes negative or 0 . This seems undesirable. ${ }^{14}$

Another issue is that QFD presupposes that all $a_{i j}$ are constant. This seems not always adequate. In the pencil example (Fig. 2), for example, a medium correlation between "easy to hold" and "length of pencil"' is presupposed. It might, however, be the case that the correlation is much stronger for rather short pencils of, say, 3-4 centimetres than for "normal'" medium-sized pencils. If the engineering characteristic is understood in the sense of "more is better', it might even be the case that above some length the correlation becomes negative because longer pencils become less easy to hold.

Presuppositions that are similar to those made with respect to $a_{i j}$ are made in QFD with respect to the tradeoffs $z_{j k}$ between the engineering characteristics. Also these presuppositions are often not realistic: not only the intensity of trade-offs between engineering characteristics may change over the domain of the engineering characteristics, even the direction-positive versus negative trade-offsmay change (Ramaswamy and Ulrich 1992).

\subsection{From customer demands to engineering characteristics}

The methodological problem attached to translating customer demand weights in weights for the engineering characteristics is deeper than the presumption that all $a_{i j}$ are non-negative and constant. It might even be doubted if this translation is possible at all.

At first sight, the step from customer demands to engineering characteristics seems less subjective than the step in which the relative importance of customer demands is determined. Engineering judgement plays an important role in this step. As Fung et al. write "The determination of...[engineering characteristics] and relation ratings depends to a great extent on the expert's knowledge of the specified product and the designer's experience', (Fung et al. 2003, p. 252).

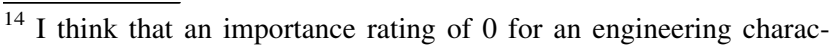
teristic might be defensible if it is plausible that what is attainted in terms of overall customer satisfaction through customer demands $a_{1}$, $a_{2}, \ldots$ by increasing the engineering characteristic target value by one unit is cancelled out by what is lost in terms of overall customer satisfaction by the effect of that same engineering characteristic on customer demands $b_{1}, b_{2}, \ldots$ A negative overall importance of an engineering characteristic may be repaired in many cases by reformulating the engineering characteristic. I think that the distinction between the first and second approach boils down to whether it is presupposed that we know beforehand what the optimal value of an engineering characteristic is. If that is presupposed negative $a_{i j}$ may make sense; if it is not, they do probably not.
}

It could reasonably be argued, I think, that usually the following determinations can more or less "objectively", be made on the basis of engineering judgement:

- The identification of which engineering characteristics have a bearing on which customer demands.

- The putting in order of importance of the engineering characteristics relevant for a certain customer demand, allowing for engineering characteristics that are ordered equally important.

These points imply that the engineering characteristics can be ordered on a weak ordinal scale with respect to their importance for the individual customer demands. Such a weak ordinal scale, however, is not enough to achieve what QFD aims at: an ordering of the importance of the engineering characteristics on the basis of their importance for a number of customer demands. This is so because the choice situation is analogous to the choice situation for which Arrow originally developed his Impossibility Theorem (cf. Franssen 2005). Arrow considered a situation in which individual preferences are to be translated into a collective preference ordering; here we have the individual preferences replaced by collective customer demands and the collective preference ordering replaced by an ordering of the engineering characteristics. ${ }^{15}$ Like in Arrow's original case, the input information is ordered on a weak ordinal scale. Like in the translation of individual customer demands into collective ones, all Arrow requirements (collective rationality, unrestricted domain, Pareto principle, independence of irrelevant alternatives, absence of a dictator) seem quite reasonable (cf. Franssen 2005).

The condition that is not met by the conventional QFD approach is that of "independence of irrelevant alternatives,' which here means that the ordering of two engineering characteristics may not depend on the inclusion or the exclusion of a third one. In the pencil example, leaving out the engineering characteristic "hexagonality" has the effect that the engineering characteristic "length of pencil', which was first the least important engineering characteristic (Fig. 3) now becomes the most important (Fig. 5), at least in terms of the technical weights.

In the light of this example, one might cast some doubts on the reasonableness of the condition "independence of irrelevant alternatives'. Given the fact that the engineering characteristics "length of pencil"' and "hexagonality" fulfil more or less the same customer demands, it seems not unreasonable that when one of these two characteristics is

\footnotetext{
${ }^{15}$ A difference is that the customer demands have different degrees of importance whereas in the original Arrow choice situation each individual has equal weight. We can, however, repair this by replacing each customer demands by $x$ customer demands where $x$ is the (relative) degree of importance of that customer demand (Franssen 2005).
} 
Fig. 5 Pencil example if the engineering characteristic

"hexagonality" is left out

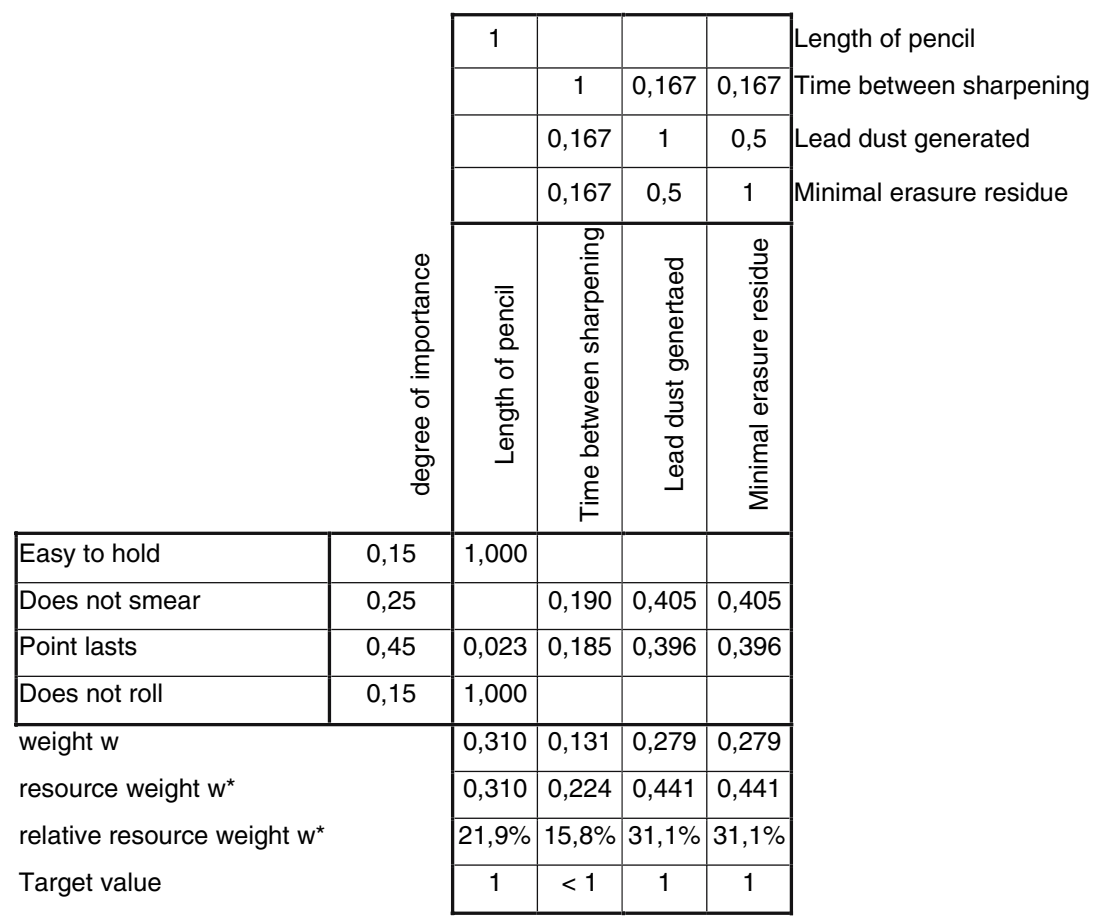

not taken into account the other becomes relatively more important. However, the fact that "length of pencil" now becomes the most important engineering characteristic would mean-if improvement efforts would first focus on the engineering characteristics with the highest technical importance-that the efforts would first focus on the two customer demands, viz. "easy to hold" and "does not roll", which are considered the least important, whereas in the original situation (Fig. 3) efforts would first focus on the other two customer demands. If we look at the resource weight of the engineering characteristics the picture is somewhat different; "length of pencil" is now the third important characteristic. However, the original target value for "length of pencil" was 0.13 (Fig. 3). In the new situation this target value would probably become 1 and the target value for "time between sharpening" would probably drop below 1. Again this implies more emphasis on the customer demands "easy to hold" and "does not roll" at the cost of the other customer demands.

Apart from what might be said about the pencil example, it does not seem desirable in general that the ordering of two engineering characteristics depends on the inclusion or exclusion of a third one. The condition "independence of irrelevant alternatives," moreover, forbids the use of non-ordinal information (Sen 1970, pp. 89-92), while QFD presuppose that the numbers in the relationship matrix can be measured on a ratio scale (cf. Otto 1995). As I have argued above, it is not very likely that such a measurement is possible. Typically, in the literature on QFD no arguments are given why or how a measurement of relationships on a ratio scale that is uniform over the various engineering characteristics and customer demands would be possible or meaningful.

\subsection{Target values}

In the approach for setting targets proposed by Bode and Fung (1998), target values are expressed as a fraction of the optimal meeting of an engineering characteristic. In many cases, however, it is not clear what this means. Take for example the engineering characteristic "lead dust generated" for a pencil. It is already difficult to determine what it would mean to optimally meet this requirement, but without some measurement scale, a target liking meeting this requirement for $70 \%$ is meaningless. It should be noted that even if a measurement scale is available for some engineering characteristics, this scale is usually not uniformly related to meeting customer demands, while that is presupposed by the formula used for customer satisfaction:

$S=\sum_{i=1}^{m} d_{i} s_{i}=\sum_{i=1}^{m} \sum_{j=1}^{n} d_{i} a_{i j} e_{j}=\sum_{j=1}^{n} w_{j} e_{j}$.

Take for example the engineering characteristic "length of pencil". This length can be expressed in centimetres. Now suppose that the optimal value is $15 \mathrm{~cm}$. What does it mean to meet this requirement by $50 \%$ ? One way to construct a measurement scale for degree of attainment is to 
calculate it as the quotient of the actual length and the optimal length $(15 \mathrm{~cm})$. So, $7.5 \mathrm{~cm}$ is equivalent to meeting this target with 50 and $20 \%$ corresponds with $3 \mathrm{~cm}$. This scaling, however, has no obvious connection to customer satisfaction. A decrease from 100 to $80 \%$ in the value of "length of pencil" might not have the same value for a customer as the decrease from 80 to $60 \%$. As can be seen from the formula above a decrease in $e_{k}$, i.e. the attainment of engineering characteristic $\mathrm{k}$, from $1(100 \%)$ to $0.8(80 \%)$ has the same impact on overall customer satisfaction as a decrease from $0.8(80 \%)$ to $0.6(60 \%)$. For the reasons outlined, this presupposition will usually not hold.

\section{Alternative QFD approaches}

A number of methodological issues in QFD that I have described have been discussed in the literature on QFD before. Arrow's theorem, for example, has been the subject of discussion since Hazelrigg argued that this theorem " $p r o v e s$ that currently popular approaches to design optimisation such as Total Quality Management (TQM) and QFD, are logically inconsistent and can lead to highly erroneous results"' (Hazelrigg 1996, p. 161). Various authors have suggested methods for dealing with this fundamental methodological problem (Scott and Antonsson 1999; Lowe and Ridgway 2000; Dym et al. 2002), which have again been criticized (Franssen 2005). Also a number of other methodological problems that I have described have drawn attention in the literature on QFD (Ramaswamy and Ulrich 1992; Wasserman 1993; Matzler and Hinterhuber 1998; Park and Kim 1998; Vairaktarakis 1999; Cook and $\mathrm{Wu} 2001)$. In several cases, this has led to proposals for improved or more sophisticated QFD methods.

In this section, I will investigate whether a number of alternative QFD approaches might be helpful in alleviating the methodological problems that I have sketched in the previous sections. I will discuss the alternative approaches under four headings. The first one covers alternative approaches that take the basic approach to QFD as I have sketched it in Sect. 2 for granted and further refine this approach. Under the second heading, I include alternative approaches, such as multi criteria analysis and pairwise comparison charts, for selecting alternatives or engineering characteristics. The third heading covers approaches that focus on market segments; the fourth deals with approaches based on demand modelling.

\subsection{Sophisticated QFD approaches}

In the literature on QFD, a whole range of more sophisticated approaches has been proposed. Some of these are intended to deal with the methodological problems I have sketched; others mainly aim at a more precise and mathematically sophisticated formulation of QFD. My aim is not to give a complete overview, but only to sketch some current developments and to indicate whether these are promising for eventually overcoming the earlier sketched methodological problems or not.

A first development is the integration of Kano's model for customer satisfaction into QFD (Matzler and Hinterhuber 1998). Kano's model makes a distinction between three types of user demands:

- Must be requirements. If these are not me, customers will be extremely dissatisfied, but these requirements do not positively contribute to perceived customer satisfaction.

- One-dimensional requirements. Customer satisfaction is supposed to be proportional to the degree to which these requirements are fulfilled.

- Attractive requirements. These are extra product features. Customers are not dissatisfied if these requirements are not met, but if these requirements are met, the rate of customer satisfaction is disproportional.

This distinction can be seen as an attempt to address the methodological problem that tradeoffs between customer demands are usually not constant (Sect. 3.2). Distinguishing between these three types of requirements helps to avoid this oversimplified assumption. Matzler and Hinterhuber (1998) propose different indexes for customer satisfaction and customer dissatisfaction for customer demands. They do, however, not offer a method for translating these into priorities among the engineering characteristics or into target values. Still, although Kano's model does not address the more fundamental methodological problems in QFD, it goes some way in addressing the issues described in Sect. 3.2.

A second development is the use of more sophisticated rating scales for the relation between customer demands and engineering characteristics. Park and Kim (1998), for example, criticize the choice of rating scales like 1-3-9. They propose to use a cardinal scale instead of an ordinal scale for these ratings, and so try to address the methodological issue discussed in Sect. 4.1. They fail to argue, however, how it would be possible to measure the correspondence between customer demands and engineering characteristics on a cardinal scale and on one that is uniform for all the different correspondences. Another proposal to deal with this methodological problem is sensitivity analysis (e.g. Shen et al. 1999). Again, this does not solve the fundamental problem. Sensitivity analysis applies different rating scales and tests whether this results in different outcomes. In doing so, it presupposes that the (relative) weight of the engineering characteristics can be expressed as a linear additive function of the (relative) 
importance of the customer demands. However, the distorting effect of this assumption might well be larger than the mere choice of the rating scale. For such reasons, the added value of sensitivity analysis is limited.

A third development is more sophisticated methods for target setting (e.g. Fung et al. 1998, 2003, Kim et al. 2000; Tang et al. 2002). Such more sophisticated methods, for example, try not simply to maximize customer satisfaction given a budget constraint, but also introduce additional constraints, for example a minimum degree to which each engineering characteristic has to be met. Some methods also try to differentiate between different types of resources instead as just overall costs. Finally, a number of approaches use fuzzy models to deal with impreciseness and uncertainty. Sophisticated as they approaches might be, they neither address the more fundamental methodological problems that are due to Arrow's Impossibility Theorem nor the more specific problems with respect to target setting I have sketched. Some in fact seem to increase the methodological problems by making more or stronger assumptions than conventional QFD approaches do.

\subsection{Alternative selection procedures}

QFD is usually not understood as a method for choosing between different design concepts, but as a method for setting engineering targets. Nevertheless, the outcomes of QFD can be used to choose between different designs. It is therefore interesting to see if alternative selection procedures exist that can help to overcome the methodological problems of QFD. In the literature, a number of approaches has been proposed that claim, sometimes implicitly, to overcome the methodological problems that arise due to Arrow's Impossibility Theorem.

Franceschini and Rossetto (1995) have proposed multi criteria analysis, in combination with outranking, as an alternative to the relationship matrix in QFD for setting the relative importance of the engineering characteristics. However, this does not overcome Arrow's Impossibility Theorem, even if Scott and Antonsson (1999) claim that multi criteria analysis is not plagued by Arrow's Impossibility Theorem. Franssen (2005), however, has shown that their arguments beg the question because they presuppose that an aggregate order among the multiple criteria exists, while that is just what is at stake.

Dym et al. (2002) have proposed pairwise comparison charts for comparing alternative designs, a method that could also be used to rank design criteria or engineering characteristics by importance. As they show, their approach is equivalent to the Borda count, i.e. it gives the same outcome. The Borda count is known to violate the condition "independence of irrelevant alternatives" of Arrow. Saari has argued that the Borda count is neverthe-
Table 2 Preferences of three groups of customers with respect to colour, size and shape

\begin{tabular}{llll}
\hline Preferences & Colour & Size & Shape \\
\hline Customer 1 & Red & Large & Flat \\
Customer 2 & Red & Small & Bumpy \\
Customer 3 & Green & Large & Bumpy \\
Collective & Red? & Large? & Bumpy? \\
\hline
\end{tabular}

less superior to others methods of aggregation because it uses all relevant available information (Dym et al. 2002). It is, however, contestable what information exactly is relevant and available (cf. Franssen 2005, p. 48).

\subsection{Market segmentation}

A third category of alternatives focuses on market segmentation. To understand the importance of market segments, it is useful to look at an example presented by Hazelrigg (1996) to show how Arrow's theorem can result in erroneous QFD results. Suppose a product has three attributes: colour, size and shape, and suppose that each attribute has two possible options: red or green (colour), large or small (size) and flat or bumpy (shape). Suppose that there are three groups of customers, whose preferences are represented in Table 2.

On the basis of this table, one might be tempted to think that the group preference is a red, large, bumpy product. It might be the case, however, that customer 1 dislikes a bumpy product so much that is has no value to him, while customer 2 dislikes large products so much that they have no value to her; for customer 3 , finally, red products may have no value at all. What seems to be the most preferred product is actually disliked by all customers.

Lowe and Ridgway (2000) present two possible solutions to the example presented by Hazelrigg. The first has to do with how the preferences of the three individuals are aggregated. Hazelrigg presupposes a kind of majority voting on each attribute separately. However, we might also ask each of the customers to rate the importance of each attribute on a scale from 0 to 1 and then calculate the weighted average importance of each attribute. Even if this procedure gives a better solution in this particular case, as Lowe and Ridgway argue, it does obviously not solve the fundamental issues that arise due to Arrow's Impossibility Theorem. ${ }^{16}$ In other situations, it might be Lowe and Ridgway's aggregation method instead of Hazelrigg's one that leads to "erroneous" results.

\footnotetext{
${ }^{16}$ In fact, this mode of aggregation presupposes interpersonal utility comparison, which is not allowed according to standards accounts of decision theory.
} 
The second solution presented by Lowe and Ridgway is to supply not a single product but a number of products, each of which would satisfy some customer group; in this case, this would imply the supply of three different products. In terms of Arrow's Impossibility Theorem, the choice of a specific customer segment can be seen as way to introduce domain restrictions, so weakening the second condition (unrestricted domain) on which the theorem rests: we only count certain customers as members of a market segment if their preferences meet certain domain restrictions.

It is known that under certain domain restrictions Arrow's Impossibility Theorem does not apply. One such a restriction is single-peakedness. This condition implies that there is one underlying criterion alongside which all the individuals order the options. An example is the left-right distinction in politics. The idea is that while individuals will prefer different options on the left-right axis, their preferences will fall monotonically to both the left and the right side of their most-preferred option on the left-right axis. A similar restriction does not seem reasonable in QFD, however. If customer preferences are eventually determined by only one criterion, what is the point in distinguishing different customer demands in QFD? This seems to presuppose that there is in fact not one underlying criterion, and there are, I think, good reasons for this presupposition.

Nevertheless, the use of market segments may introduce domain restrictions that even if they do not avoid Arrow's Impossibility Theorem, at least alleviate the consequences of it. It can be shown, for example, that under reasonable domain restrictions for market segments, QFD results in a Pareto improvement among the customers in that market segment, so avoiding the erroneous results suggested by Hazelrigg.

To show this, I start with supposing that the preferences of each customer in a market segment can be represented by an ordinal value function. This is usually possible if the preferences of each customer over the options form a weak order. Note that in this case, the options are formed by some combinations of the values of the customer demands $s_{i}$ as discussed in Sect. 3.2. In contrast to there, I do not presuppose that the value function takes the form of a linear additive value function. This supposition can be written as: Now also suppose that the value function $v_{x}$ of each customer $\mathrm{x}$ in market segment $m$ has the following two properties: These conditions could be used to define market segments, so that these conditions are by definition met in each market segment. (Note that in different market segments $s_{i}, \ldots, s_{m}$ can be different). In this situation, an improved meeting of the customer demands will result in a Pareto improvement, in the sense that no customer in the market segment is worse off and at least one customer is better off.

Now suppose that it is also possible to find a set of engineering characteristics meeting the following conditions:
1. For each customer $x$, a value function $v_{x}$ of customer demands $s_{i}, \ldots, s_{m}$ exist so that $v_{x}\left(a_{i}, \ldots, a_{m}\right) \geq$ $v_{x}\left(b_{i}, \ldots, b_{m}\right) \Leftrightarrow \mathbf{a}\left(a_{i}, \ldots, a_{m}\right) \succsim \mathbf{b}\left(b_{i}, \ldots, b_{m}\right)$ where $a_{i}, \ldots, a_{m}$ and $b_{i}, \ldots, b_{m}$ are different combinations of values for $s_{i}, \ldots, s_{m}$ and $\mathbf{a} \succsim \mathbf{b}$ means that customer $x$ weakly prefers option $a$ over option $b$.

2. $d v_{x} / d s_{i} \geq 0$ for all customer demands $s_{i}$ of each customer $x$.

3. $d v_{x} / d s_{i}>0$ for at least one combination of $i$ and $x$.

4. For each customer demand $s_{i}$ an ordinal value function $\gamma_{i}$ of the engineering characteristics $e_{j}, \ldots, e_{n}$ exist so that $\gamma_{i}\left(x_{j}, \ldots x_{n}\right) \geq \gamma_{i}\left(y_{j}, \ldots, y_{n}\right) \Leftrightarrow x\left(x_{j}, \ldots x_{n}\right) \succ y\left(y_{j}, \ldots, y_{n}\right)$ where $x_{j}, \ldots, x_{n}$ and $y_{j}, \ldots, y_{n}$ are different combinations of values for $e_{j}, \ldots, e_{n}$ and $\mathbf{x} \succsim_{i} \mathbf{y}$ means that option $x$ meets customer demand $s_{i}$ at least as good as option $y$.

5. For each customer demand $s_{i}: \frac{d \gamma_{i}}{d e_{j}} \geq 0$ for all engineering characteristics $e_{j}$.

6. $\frac{d \gamma_{i}}{d e_{j}}>0$ for at least one combination of $i$ and $j$.

If conditions 4,5 and 6 are met, better meeting one of the engineering characteristics-without doing worse on any of the other engineering characteristics-automatically implies a Pareto improvement among the customer demands. If conditions 1,2 and 3 are also met, this also implies a Pareto improvement for the customers.

Note that in traditional QFD, it is presupposed that $\gamma_{i}$ can be written as

$\gamma_{i}=\sum_{j=1}^{n} a_{i j} e_{j}$ with $a_{i j} \geq 0$.

Under this presupposition, conditions 4,5 and 6 are indeed met. Note, however, that conditions 4, 5 and 6 are much weaker than what is usually presupposed in QFD. It is, for example, not presupposed that each $\gamma_{i}$ can be written as a linear additive value function of $e_{j}$, so avoiding a number of the methodological problems discussed in Sect. 4.1. (Note, however, that condition 5 and 6 are a kind of reformulation of the presupposition in QFD that $a_{i j}$ is always non-negative.) It should also be noted that condition 4 is not plagued by Arrow's Impossibility Theorem. The reason is that condition 4 requires the solution of a single criterion instead of a multiple criteria problem. ${ }^{17}$ Condition 4 requires that it is possible to weakly order different combinations of engineering characteristics $e_{j}, \ldots, e_{n}$ with

\footnotetext{
${ }^{17}$ The ordinal value function $\gamma_{i}$ could be conceived of as a representation of the subjective judgment ("preferences") of an expert over combinations of engineering characteristics $e_{j}, \ldots, e_{n}$ with respect to one specific customer demand $s_{i}$. This supposes that the expert is able to solve the single criterion problem in a generally acceptable way. I argued for the likeliness of this assumption in Sect. 4.2. Alternatively, it might be possible to construct a value function $\gamma_{i x}$ for each customer $x$. Each of these value functions then have to meet condition 4 and 5, and at least one $\gamma_{i x}$ has to meet condition 6 .
} 
respect to one specific customer demand $s_{i}$; it does not require weakly ordering combinations of engineering characteristics $e_{j}, \ldots, e_{n}$ with respect to combinations of customer demands $s_{i}, \ldots, s_{m}$. So, we only have one "voter", i.e. customer demand $i$, while Arrow's Impossibility Theorem only applies to two or more "voters".

Market segmentation can thus be used to introduce certain domain restrictions. A minimal result that can be achieved by market segmentation is to avoid that some customers are actually less satisfied with the new product than with the current product. It might be possible to introduce even stricter, but still plausible, domain restrictions than those suggested above in defining market segments, so that Arrow's theorem can be avoided. ${ }^{18}$ This is, however, beyond the scope of this article.

\subsection{Demand modelling}

Another interesting development is demand modelling to predict the demand for products with certain features. On the basis of such predictions, the desirable characteristics of products can be chosen. Some authors have also attempted to introduce such considerations into QFD. I will discuss at some length a proposal developed by Cook and Wu (2001).

Cook and $\mathrm{Wu}$ use the so-called S-model to predict user demand. This is a phenomenological model for predicting demand, expressed in terms of the values and prices of products. The demand is taken to be equal to the total amount of a product sold over a period of time, assuming that there is no scarcity of supply. The value of a product is a measure for the amount of money people are willing to spend on that product. The assumption is that people buy a product if its value is higher than its price. If demands and prices are known for a range of competing products, the value of these products can be calculated.

Using the S-model, predictions of future demand can be made if prices and values of a new product are known. The difficult part, of course, is to predict the value of a new product. Cook and $\mathrm{Wu}$ propose the direct value (DV) method to this end. In this method, customers are asked to compare a baseline product with an imaginary alternative product in which one or more of the values of the product attributes have been modified. The customers are asked to choose between the baseline and the alternative product for a series of prices of the alternative product. Next, the fraction of respondents choosing the alternative is plotting

\footnotetext{
18 See Sen (1970, Chapt. 10*) for domain restrictions under which Arrow's Impossibility Theorem does not apply. None of the restrictions is however prima facie plausible for product development or market segments.
}

against the price of the alternative. On basis of this plot, a so-called neutral price $P_{\mathrm{N}}$ is determined; this is the price at which half of the respondents chooses the alternative product and half the baseline product. On basis of the $\mathrm{S}$-model, it can now be shown that:

$V-V_{0}=P_{\mathrm{N}}-P_{0}$

In this formula $V_{0}$ is the value of the baseline product and $P_{0}$ is the price of the baseline product. $V$ is the value of the new product. If $V_{0}$ and $P_{0}$ are known, and $P_{\mathrm{N}}$ has been determined, $V$ can easily be calculated.

The DV method is usually used for one attribute change at the time. Note that this supposes that the attributes are preferentially independent: the change in value due to changes in one attribute does not depend on the values of the other attributes. As discussed earlier, this might be a problematic assumption (Sect. 3.2). The DV method also presupposes that people can compare non-existing products with current ones, which might be problematic (cf. Sect. 3.1).

Another methodological issue with respect to the DV method is that, from earlier research, it is known that there is a gap between the maximum price for which someone is willing to buy a product and the minimum price for which that person is willing to sell it. Most people want a higher minimum price for selling a product than they are prepared to pay for buying the same product. Usually this phenomenon is phrased in terms of Willingness to Pay (WTP) versus Willingness to Accept (WTA). Cook and Wu interpret this phenomenon in terms of uncertainty. Even if this would be a right interpretation, an implication seems to be that the DV method will probably yield different values for the same product if different baselines are chosen. In general, it might seems reasonable to use the current product as baseline; however, in reality consumers will not choose between the current product and the new product but between a number of-new-products of competitors and the newly developed product.

Cook and $\mathrm{Wu}$ integrate the S-model in QFD in order to increase the profit of the company. Their proposed QFD approach proceeds as follows. Customer demands are listed and related to engineering characteristics. The engineering characteristics for the current (baseline) product are measured. A range of alternative products is devised with other engineering characteristics. With the DV method, the changes in customer value for these alternative products are measured. Using the S-model, the expected additional demand given a certain price for the alternatives is calculated. By also estimating the expected additional costs for developing and producing each alternative, the added profit for each alternative can easily be calculated. The option with the highest additional profits is chosen. 
Some people would probably argue that the approach proposed by Cook and $\mathrm{Wu}$ is no longer a QFD approach. It does, for example, not set target values for the engineering characteristics. Although, they use a kind of House of Quality, they do not make any of the calculations mentioned in Table 1. However, by not making these calculations they avoid most of the methodological problems that were discussed in Sect. 3.3 and in Sect. 4. This is not to say that their approach is completely without methodological problems; some of these have been indicated above.

What is perhaps most important is that Cook and Wu's approach avoids Arrow's Impossibility Theorem because it does not try to aggregate individual preferences into collective ones, but just models how many people would probably buy a product with certain features. This seems more generally true for demand modelling approaches. Wassenaar et al. (2005), for example, write about their demand modelling approach that it "aggregates the customer choices (not preferences) by summing the choice probabilities across individual decision makers (customers), thus avoiding the paradox associated with aggregating the utility or preference of a group of customers" (Wassenaar et al. 2005, p. 522).

\section{Conclusions}

QFD is a potential tool for enhancing the competitiveness of companies that helps them to focus on customer demands in product development. QFD is, however, beset with a range of methodological problems. The most important of these problems are:

1. Customer demands are product dependent.

2. Customer demands cannot always be represented by a linear additive value function.

3. Individual customer preferences cannot be aggregated into a collective customer preference ordering without violating a number of very reasonable conditions.

4. The correlation between customer demands and engineering characteristics is not always non-negative and constant.

5. The relative importance of customer demands cannot be uniformly translated into a relative importance of the engineering characteristics.

6. The meaning of target values is unclear or disputable.

Of these, the third and fifth are probably the worst for QFD because they suggest that the core idea of the QFD approach is methodologically problematic. Both are due to Arrow's Impossibility Theorem and this theorem is a major obstacle for a methodologically sound QFD approach.

I have discussed a number of alternative approaches to QFD. We have seen that most of the sophisticated QFD approaches and alternative selection procedures do not help in solving the methodological issues in QFD, some of them even make the methodological problems worse. We have also seen three contributions that can be helpful in at least alleviating some methodological problems: the inclusion of Kano's model in QFD, market segmentation and demand modeling.

The inclusion of Kano's model might help to overcome methodological problem 2 at least partly. Kano's model, however, seems hard to integrate in an adequate way into a quantitative QFD approach.

Market segmentation might help to alleviate the worst consequences of Arrow's Impossibility Theorem for QFD. It might, at least, be possible to define market segments in such a way that the use of QFD implies a Pareto improvement among the customers of a product. An interesting topic for further research is whether it would be possible to use market segmentation to introduce domain restrictions under which Arrow's theorem does not apply. If that would be possible, methodological problem 3 could be solved by market segmentation, but this is still a very big if.

Alternatively, one could be satisfied with achieving a Pareto improvement with QFD and choose an approach to QFD in which one does not calculate the relative importance of customer demand and engineering characteristics and sets no target values. This would solve most methodological problems but obviously against a certain price: many would feel that improving customer satisfaction is just not good enough. I would like to stress, however, that such a qualitative QFD approach might still have a considerable added value to traditional product development approaches. For one thing, systematically looking at customer demands, engineering characteristics and their relations (positive or negative) already makes the product development process more customer-oriented. Moreover, a qualitative approach to QFD might still provide an important platform for exchange between the engineering and the marketing department within a production company and so improve product development.

Another interesting direction for the further development of QFD is demand modeling. We have seen that this approach avoids Arrow's Impossibility Theorem (methodological problem 3 ) because it does not try to aggregate individual customer preferences but just models the expected demand of a product. It also avoids methodological issue 4, 5 and 6 but simply because it does not make these kinds of calculations. As a result, demand modeling does not result in importance ratings or targets for the engineering characteristics, which may be concerned a disadvantage. Demand modeling also does not solve methodological issue 1 and 2; at least the S-model does not. Future development of demand modeling might lead to models that help solve these issues. 
It should be noted that demand modeling replaces the original QFD goal of "maximizing customer satisfaction within certain constraints" by "maximizing company profits." This might be considered a more straightforward approach because maximizing customer satisfaction is usually seen as instrumental to maximizing company profit. Nevertheless, it might be worthwhile to keep a focus on customer satisfaction apart from increasing company profits in the short run. One reason is that sometimes profits might be raised while customers are dissatisfied. In the long run, this is usually not in the interest of the company. So, one might want to ensure that new products at least increase customer satisfaction, for example by market segmentation.

This article thus suggests a number of useful directions for the further development of QFD and research on QFD. It also suggests, however, that the further sophistication of existing QFD approaches without paying attention to the current methodological problems in QFD is a non-starter.

\section{References}

Akao Y (ed) (1990) Quality function deployment. Integrating customer requirements into product design. Productivity Press, Cambridge, Mass

Arrow KJ (1950) A difficulty in the concept of social welfare. J Polit Econ 58:328-346

Bergquist K, Abeysekera J (1996) Quality function deployment (QFD): a means for developing usable products. Int J Ind Ergon 18(4):269-275

Bode J, Fung RYK (1998) Cost engineering with quality function deployment. Comput Ind Eng 35(3-4):587-590

Cook HE, Wu A (2001) On the valuation of goods and selection of the best design alternative. Res Eng Des 13:42-54

Dym CL, Wood WH, Scott MJ (2002) Rank ordering engineering designs: pairwise comparison charts and Borda counts. Res Eng Des 13:236-242

Fishburn PC (1970) Utility theory for decision making. Wiley, New York

Franceschini F, Rossetto S (1995) QFD: The problem of comparing technical/engineering design requirements. Res Eng Des 7:270278

Franssen M (2005) Arrow's theorem, multi-criteria decision problems and multi-attribute preferences in engineering design. Res Eng Des 16:42-56

French S (1988) Decision theory. An introduction to the mathematics of rationality. Ellis Horwood, New York

Fung RYK, Popplewell K, Xie J (1998) An intelligent hybrid system for customer requirements analysis and product attribute targets determination. Int J Prod Res 36(1):13-34

Fung RYK, Tang J, Yu Y, Wang D (2002) Product design resource optimization using a non-linear fuzzy quality function deployement model. Int J Prod Res 40(3):585-599

Fung RYK, Tang J, Yiliu Tu P, Chen Y (2003) Modelling of quality function deployment planning with resource allocation. Res Eng Des 14:247-255
Govers CPM (1996) What and how about quality function deployment (QFD). Int J Prod Econ 46-47:575-585

Hauser JR, Clausing D (1988) The House of Quality. Harv Bus Rev 66(3):63-73

Hazelrigg GA (1996) The implications of Arrow's Impossibility Theorem on approaches to optimal engineering design. J Mech Des 118:161-164

Keeney RL, Raiffa H (1993) Decisions with multiple objectives: preferences and value tradeoffs. Cambridge University Press, Cambridge (England); New York, NY, USA

Kim K-J, Moskowitz H, Dhingra A, Evans G (2000) Fuzzy multicriteria models for quality function deployment. Eur $\mathrm{J}$ Oper Res 121(3):504-518

King B (1989) Better designs in half the time. Implementing quality function deployment in America. Goal/QPC, Methuen

Krantz DH, Luce RD, Suppes P, Tversky A (1971) Foundations of measurement. Academic, San Diego

Lowe AJ, Ridgway K (2000) Optimization impossible? Qual Prog 33(7):59-64

Matzler K, Hinterhuber HH (1998) How to make product development projects more successful by integrating Kano's model of customer satisfaction into quality function deployment. Technovation 18(1):25-38

Otto KN (1995) Measurement methods for product evaluation. Res Eng Des 7:86-101

Park T, Kim K-J (1998) Determination of an optimal set of design requirements using House of Quality. J Oper Manage 16(5):569_ 581

Pugh S (1991) Total design: integrated methods for successful product engineering. Addison-Wesley Pub. Co., Wokingham, England; Reading, Mass

Ramaswamy R, Ulrich K (1992) Augmenting the House of Quality with engineering models. In: Design Theory and Methodology - DTM '92 - 4th international conference on Design Theory and Methodology, ASME, Scottsdale, Arizona, pp 309-316

Sarlemijn A, Boddendijk HG (eds) (1995) Produkten op maat. QFD als gids bij produktcreaties. Boom, Amsterdam, Meppel

Scott MJ, Antonsson EK (1999) Arrow's theorem and engineering design decision making. Res Eng Des 11:218-228

Sen AK (1970) Collective choice and social welfare. Oliver \& Boyd, Edinburg and London

Shen XX, Tan KC, Xie M, Goh TN, Wang H (1999) Sensitivity of the relationship matrix in quality function deployment. Int J Ind Eng Theory Appl Pract 6(3):214-223

Shullito ML (1994) Advanced QFD. Linking technology to market and company needs. Wiley, New York

Tang J, Fung RYK, Xu B, Wang D (2002) A new approach to quality function deployment planning with financial considerations. Comput Oper Res 29:1447-1463

Vairaktarakis GL (1999) Optimization tools for design and marketing of new/improved products using the house of quality. J Oper Manage 17(6):645-663

Van Gorp A (2005) Ethical issues in engineering design. Safety and sustainability. Simon Stevin Series in the Philosophy of Technology, Delft

Wassenaar HJ, Chen W, Cheng J, Sudjianto A (2005) Enhancing discrete choice modeling for decision-based design. Trans ASME 127:514-522

Wasserman GS (1993) On how to prioritize design requirements during the QFD planning process. IIE Trans 25(3):59-65

Xie M, Goh TN, Wang H (1998) Study of the sensitivity of "customer voice' in QFD analysis. Int J Ind Eng Theory Appl Pract 5(4):301-307 